\title{
Interspike-interval correlations induced by two-state switching in an excitable system
}

\author{
Tilo Schwalger ${ }^{1(a)}$, Jordi Tiana-Alsina ${ }^{2(a)}$, M. C. Torrent ${ }^{2}$, Jordi Garcia-Ojalvo ${ }^{2(b)}$ \\ and BENJAMIN LINDNER ${ }^{1}$ \\ 1 Bernstein Center for Computational Neuroscience and Institut für Physik, Humboldt Universität zu Berlin \\ Philippstr. 13, 10115 Berlin, Germany, EU \\ ${ }^{2}$ Departament de Física i Eng. Nuclear, Universitat Politècnica de Catalunya - Edif. GAIA, \\ Rambla de Sant Nebridi s/n, 08222 Terrassa, Barcelona, Spain, EU
}

received 22 March 2012; accepted in final form 14 June 2012

published online 10 July 2012

PACS 05.45.-a - Nonlinear dynamics and chaos

PACS 05.10.Gg - Stochastic analysis methods (Fokker-Planck, Langevin, etc.)

PACS 42.60.Mi - Dynamical laser instabilities; noisy laser behavior

\begin{abstract}
We study correlations of intervals between pulses in an excitable system, using a semiconductor laser with optical feedback as an experimental model system. First we show, by means of a combination of experimental observations and theoretical analysis, that for an intermediate range of the laser's pump current the interspike intervals are positively correlated over a few lags, an effect that can be theoretically explained by an intrinsic two-state switching in the laser dynamics. The same theory can be also applied if the laser is externally driven by a dichotomous switching of the pump current, a scenario that allows for a controlled change of the spike rates of the two states over orders of magnitude. Varying one of the pump levels, we find experimentally that the correlation between adjacent intervals is maximized at a finite pump level corresponding to an optimal ratio of dropout rates in the two states. Our theory confirms these findings and reveals how the regularity of spiking in the two states shapes the correlation maximum.
\end{abstract}

Copyright (C) EPLA, 2012

The behavior of various natural and technological systems often takes the form of sequences of discrete events (point processes), whose statistical properties can be controlled both by the internal dynamics of the system and by the environmental conditions to which the system is subjected. Correlations in the time intervals between subsequent events (named interspike intervals, ISIs, in what follows) arise in geophysics (earthquakes) [1], laser physics $[2,3]$, and neuroscience $[4,5]$, among other fields. ISI correlations can be functionally relevant, as in sensory neurons, in which they are known to influence information transfer [6] by affecting low-frequency noise [7]; they may also betray the dominant noise source in cells [8]. Standard excitable models such as the FitzHugh-Nagumo model driven by fast fluctuations generate approximately independent ISIs (i.e. renewal processes in which ISI correlations vanish).

\footnotetext{
(a) These authors equally contributed to this paper.

(b) E-mail: jordi.g.ojalvo@upc.edu
}

In this paper, we provide experimental evidence of non-renewal behavior in an excitable system, namely a semiconductor laser with optical feedback, and show that ISI correlations in this system are consistent with a stochastic switching between different sub-states. For the case of two-state switching, we derive an expression for the correlation coefficient that matches the experimental data quantitatively, for either internally or externally evoked correlations. In particular, external control over the two-state switching (implemented experimentally via a dichotomous modulation of the laser's pump current) reveals that the correlation in the sequence of interspike intervals is maximized at a certain value of the ratio of dropout rates, in agreement with the theoretical prediction.

In our experimental setup, the laser is subject to optical feedback through an external mirror, which leads (provided the feedback strength is moderate and the pump current is close to threshold) to an irregular series of power dropouts (our "spikes" in what follows), as shown 
(a)

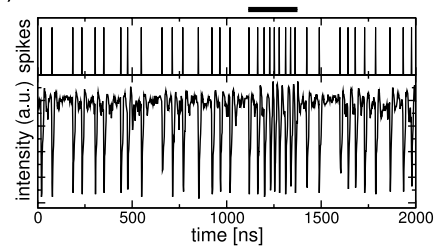

(b)

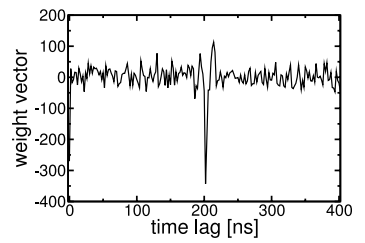

Fig. 1: (a) Laser output for a constant pump current (lower panel, 32.2 mA) and detected spikes (upper panel); the black horizontal bar indicates a region of increased dropout rate. (b) Weight vector $\vec{w}$ used for used spike/no spike classification.

in fig. 1(a). Even though this behavior is the result of delay-induced instabilities and is thus high-dimensional in nature $[9,10]$, experimental and theoretical studies have shown that the power dropouts can be interpreted as excitable pulses $[11,12]$, which have even been reproduced with low-dimensional models [13]. Therefore, here we use this system as a test bed to study the emergence of interspike interval correlations in generic excitable systems.

The laser used in our experiment is an AlGaInP FabryPerot semiconductor laser, operating at a nominal wavelength around $650 \mathrm{~nm}$. The laser intensity is captured by a high-speed fiber photodetector with $2 \mathrm{GHz}$ bandwidth. The signal is then amplified, using a $2-\mathrm{GHz}$ high-speed electronic amplifier, and sent to an oscilloscope with $1 \mathrm{GHz}$ bandwidth. In order to modulate the pump of the laser we use a $100 \mathrm{kHz}$ Bias T. Figure 1(a) shows the dynamics of the laser in response to a constant DC input in its pump current. The feedback strength and the pump level are chosen such that the laser operates in the above-mentioned power-dropout regime.

For the general purpose of this study, spike detection by threshold crossing is insufficient. Although spikes are in all considered cases easily recognizable by eye, in particular, for the laser with a dichotomous pumping current one obtains superimposed large transients, which make a spike detection by simple threshold crossing difficult. An additional complication arises because spikes differ significantly in their amplitude depending on the value of the pumping current. For these reasons, power dropouts have been detected based on their shape using a pattern classification algorithm. We used a perceptron and further conditions on the local statistics of the laser intensity to decide whether the intensity time series in a given time window has spike shape (quantified by the weight vector $\vec{w}$ shown in fig. $1(\mathrm{~b})$ ) or not. Specifically, we go over the laser-intensity time series with a sliding time window of $400 \mathrm{~ns}$, yielding a $n=201$-dimensional vector $\vec{x}$ (time step $\Delta t=2 \mathrm{~ns}$ ). The vector $\vec{x}$ is then rescaled and shifted to cover the interval $[0,1]$. The perceptron rule is: If $\vec{w} \cdot \vec{x}<\theta$, where $\theta$ is a threshold, the pattern is classified as "no spike". If $\vec{w} \cdot \vec{x}>\theta$ and additional conditions (see below) are matched, we assign a spike to the central time instant and shift the time window forward by a refractory

(a)
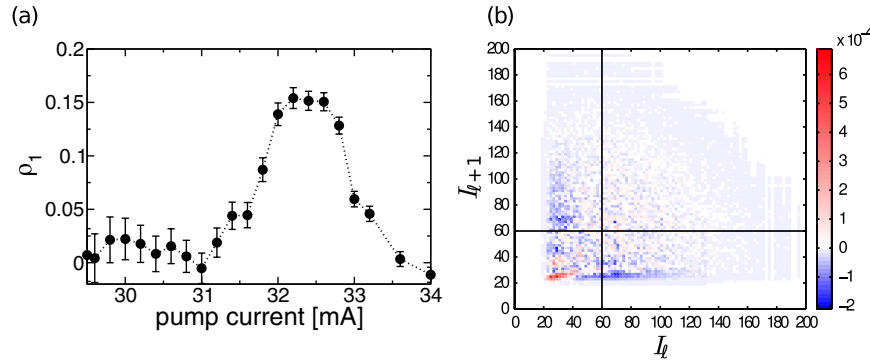

Fig. 2: (Colour on-line) (a) Correlation coefficient of adjacent intervals $\rho_{1}$ for different levels of the pump current. (b) Correlation map $\delta P\left(I_{\ell}, I_{\ell+1}\right)$ of the DC data for a pump current of $32.2 \mathrm{~mA}$; solid lines indicate the mean interval. Here and in all other figures, the vertical error bars of the SCC are calculated as in [14].

time of $14 \mathrm{~ns}$; for any "no-spike" result, the shift is only $\Delta t$. Weight vector $\vec{w}$ and threshold $\theta$ were determined using the perceptron learning rule on a training set of 169 "spike" and 127 "no spike" examples. Additional conditions for acceptance of a spike: i) $(1 / n) \sum_{i} x_{i}>0.6$; ii) the skewness of $x_{i}$ had to be smaller than -0.8 ; and iii) the local standard deviation (averaged over 10 points surrounding the suspected spike) had to be larger than 0.2 . The specific parameter values of the classification algorithm were chosen in order to optimize detection for a broad range of pump currents.

Once the spikes are detected, we examine how they are organized in time. To this end, we define the $\ell$-th time interval between consecutive dropouts as $I_{\ell}$, and calculate the correlation between intervals separated by $n$ dropouts as

$$
\rho_{n} \equiv \frac{\left\langle I_{\ell+n} I_{\ell}\right\rangle-\left\langle I_{\ell+n}\right\rangle\left\langle I_{\ell}\right\rangle}{\left\langle I_{\ell}^{2}\right\rangle-\left\langle I_{\ell}\right\rangle^{2}} .
$$

By definition $\rho_{0}=1$, while $\rho_{1}$ measures the level of correlation between adjacent intervals. For a renewal process (e.g., a Poisson process) intervals are independent of each other and $\rho_{n}=0$ for $n>0$.

As depicted in fig. 2(a), the spike sequence is uncorrelated up to a pump level around $31 \mathrm{~mA}$, beyond which the intervals between spikes appear to be significantly correlated. The coefficient $\rho_{1}$ at constant pumping becomes maximum at about $32 \mathrm{~mA}$, and for sufficiently high pumping (still in the power-dropout regime) the correlations start to decrease until reaching zero again for a pump level $\sim 34 \mathrm{~mA}$.

In order to investigate the origin of these correlations, we plot in fig. 2(b) the difference $\delta P\left(I_{\ell}, I_{\ell+1}\right)$ between the joint ISI probability density $P\left(I_{\ell}, I_{\ell+1}\right)$ of the return map, i.e. of adjacent ISIs, and the value corresponding to a renewal process, $P\left(I_{\ell}\right) P\left(I_{\ell+1}\right)$, where $P\left(I_{\ell}\right)$ is the single ISI density [15]. The plot shows an excess of short-short interval pairs $\left(I_{\ell}, I_{\ell+1}\right)$ compared to the renewal assumption, which is apparent from the significantly positive spot in the lower left corner. The short-short events are mainly related with the appearance 
(a)

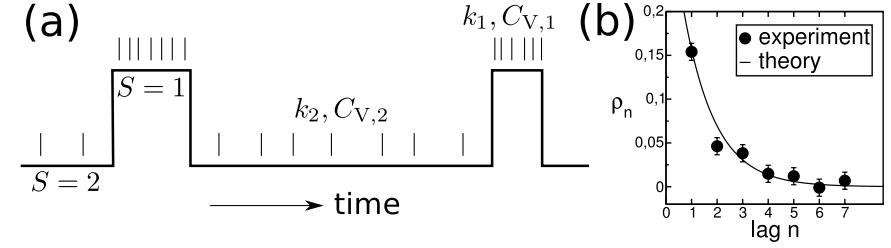

Fig. 3: (a) Sketch of the kinetic model used here, which alternates between two renewal point processes depending on the dichotomous Markov process $S(t)$. Events of the composite point process are indicated by small vertical bars. The spiking probabilities per unit time are $k_{1}$ and $k_{2}$ in the states $S=1$ and $S=2$, respectively. The ISI variability is characterized by the coefficients of variation $C_{\mathrm{V}, 1}$ and $C_{\mathrm{V}, 2}$. (b) Correlation as a function of the lag $k$ for a constant pump level of $32.2 \mathrm{~mA}$. Symbols represent the experimental data, and the solid line the theoretical fit (eq. (10)). Parameter values: $\lambda_{1}=21.936 \mathrm{MHz}, \lambda_{2}=2.654 \mathrm{MHz}, k_{1}=34.466 \mathrm{MHz}$, $k_{2}=14.823 \mathrm{Mhz}, C_{V, 1}=0.123, C_{V, 2}=0.359$.

of fast-dropout activity in the temporal series of the laser (cf. fig. 1(a)), as previously reported [13]. The combination of fast- and slow-dropout states suggests the presence of slowly changing processes for a constant pump level.

So far we have seen that the time intervals between consecutive dropouts exhibited by semiconductor lasers with optical feedback can display statistical correlations $[2,3]$ for intermediate pump levels (fig. 2(a)). We have also pointed out that the data (fig. 2(b)) suggest an intrinsic slow modulation of the rate at which the power dropouts occur. We now describe briefly a theoretical analysis of a general two-state model that links these two observations, showing that spontaneous switching between two firingrate regimes in a generic excitable medium gives rise to correlations in the corresponding ISI series.

We consider a discrete kinetic model characterized by a Markovian telegraph noise $S(t) \in\{1,2\}$ that switches stochastically between two renewal processes (see [16] for a related Markovian approximation scheme) with rates $\lambda_{1}(1 \rightarrow 2)$ and $\lambda_{2}(2 \rightarrow 1)$. The dynamics is illustrated in fig. 3(a): one state $(S=1)$ with dropout rate $k_{1}$ and coefficient of variation $C_{V, 1}$ and another state $(S=2)$ with $k_{2}$ and $C_{V, 2}$. The mean $\mu_{1 / 2}$ and variance $\sigma_{1 / 2}^{2}$ of the ISIs in the respective states are thus given by

$$
\mu_{1}=\frac{1}{k_{1}}, \quad \mu_{2}=\frac{1}{k_{2}}, \quad \sigma_{1}^{2}=\mu_{1}^{2} C_{V, 1}^{2}, \quad \sigma_{2}^{2}=\mu_{2}^{2} C_{V, 2}^{2} .
$$

Note, that our results do not depend on higher-order ISI moments. By our assumption of a Markovian telegraph process, the waiting times in each state are exponentially distributed with mean values $\lambda_{1}^{-1}$ and $\lambda_{2}^{-1}$, respectively. Note that at the transition between two states an interval can belong to both of them, and its statistics is, in general, determined by the specifics of the underlying dynamics. In our approximation, however, this boundary effect is neglected.

In order to calculate the auto-correlation with respect to the index $\ell$ of the intervals $I_{\ell}$ (and not with respect to time $t$ ), we first change to a continuous index $x$ that coincides with $\ell$ at the end of the $\ell$-th interval. Then, we can consider the state $S$ as a function of the continuous index: $S(x)$ also constitutes a random telegraph process, but with switching rates $\lambda_{1} / k_{1}$ and $\lambda_{2} / k_{2}$, respectively. Here, we used the fact that the waiting "times" in each state are approximately exponentially distributed with a mean value equal to the mean spike count $\left\langle n_{s}\right\rangle=$ $k_{s} / \lambda_{s}$ in state $s \in\{1,2\}$ (averaged over the exponentially distributed durations of state $s$ ).

The expression for the correlation coefficient can be understood as one involving two averages; one with respect to the renewal process for a fixed realization of $S(x)$ and another one with respect to the ensemble of realizations of $S(x)$. For a fixed realization of $S(x)$ all intervals are only correlated through the dichotomous switchings of $S(x)$, because of the renewal property of the single states -in particular, $\left\langle I_{\ell} I_{\ell+k}\right\rangle=\mu_{S(\ell)} \mu_{S(\ell+k)}$ for $k \geqslant 1$. The remaining average over $S(x)$ yields

$$
\begin{aligned}
\left\langle I_{\ell}\right\rangle & =\sum_{s=1,2} \mu_{s} p_{\text {st }}(s) \\
\left\langle I_{\ell} I_{\ell+n}\right\rangle & =\sum_{s, s^{\prime}=1,2} \mu_{s} \mu_{s^{\prime}} p\left(s^{\prime}, n ; s, 0\right), \quad n \geqslant 1 \\
\left\langle I_{\ell}^{2}\right\rangle & =\sum_{s=1,2}\left(\sigma_{s}^{2}+\mu_{s}^{2}\right) p_{\text {st }}(s) .
\end{aligned}
$$

Here, $p_{\mathrm{st}}(s)=\lambda_{3-s} k_{s} /\left(\lambda_{1} k_{2}+\lambda_{2} k_{1}\right)$ are the stationary probabilities of $S$ and $p\left(s, x ; s^{\prime}, x^{\prime}\right)$ is the joint probability distribution of $S(x)$ and $S\left(x^{\prime}\right)$, with $x>x^{\prime}$. The latter is equal to $p\left(s, x \mid s^{\prime}, x^{\prime}\right) p_{\text {st }}\left(s^{\prime}\right)$, where the transition probability $p\left(s, x \mid s^{\prime}, x^{\prime}\right)$ is given by $[17]$

$$
\begin{aligned}
p\left(s, x \mid s^{\prime}, x^{\prime}\right)= & p_{\mathrm{st}}(s)+\left[\delta_{s, s^{\prime}} p_{\mathrm{st}}(3-s)-\delta_{s, 3-s^{\prime}} p_{\mathrm{st}}(s)\right] \\
& \times \exp \left[-\left(\frac{\lambda_{1}}{k_{1}}+\frac{\lambda_{2}}{k_{2}}\right)\left(x-x^{\prime}\right)\right] .
\end{aligned}
$$

Specifically, using eqs. (2)-(6) we obtain the explicit expressions

$$
\left\langle I_{\ell}\right\rangle=\frac{\lambda_{1}+\lambda_{2}}{\lambda_{1} k_{2}+\lambda_{2} k_{1}}
$$

$$
\left\langle I_{\ell} I_{\ell+n}\right\rangle=\frac{\lambda_{1} \lambda_{2}\left(\lambda_{1}-\lambda_{2}\right)^{2}}{k_{1} k_{2}\left(\lambda_{1} k_{2}+\lambda_{2} k_{1}\right)^{2}} \exp \left[-\left(\frac{\lambda_{1}}{k_{1}}+\frac{\lambda_{2}}{k_{2}}\right) n\right]
$$

$$
\left\langle I_{\ell}^{2}\right\rangle=\frac{\lambda_{1} k_{1}\left(1+C_{V, 2}^{2}\right)+\lambda_{2} k_{2}\left(1+C_{V, 1}^{2}\right)}{k_{1} k_{2}\left(\lambda_{1} k_{2}+\lambda_{2} k_{1}\right)} .
$$

Substituting these expressions into the definition of the serial correlation coefficient, eq. (1), yields for $n \geqslant 1$

$$
\rho_{n}=\frac{\exp \left[-\left(\hat{\lambda}_{1}+\frac{\hat{\lambda}_{2}}{\gamma}\right) n\right]}{1+\frac{\gamma \hat{\lambda}_{1}+\hat{\lambda}_{2}}{(1-\gamma)^{2} \hat{\lambda}_{1} \hat{\lambda}_{2}}\left(\gamma \hat{\lambda}_{2} C_{V, 1}^{2}+\hat{\lambda}_{1} C_{V, 2}^{2}\right)}
$$

with $\gamma=k_{2} / k_{1}, \hat{\lambda}_{1}=\lambda_{1} / k_{1}, \hat{\lambda}_{2}=\lambda_{2} / k_{1}$. 
To test whether the serial correlations of the laser dropouts can be indeed associated to two alternating renewal processes, we estimated the parameters of the twostate model from the experimental sequence of ISIs. To this end, we labeled each interval in the experimental time series as "fast"- or "slow"-dropout state. This was decided based on whether the ISI was smaller or larger than a threshold of $35 \mathrm{~ns}$. The value of this threshold was chosen because the lines $I_{\ell}=35 \mathrm{~ns}, I_{\ell+1}=35 \mathrm{~ns}$ enclose the excess region of short-short pairs in fig. $2(\mathrm{~b})$, that is associated with the fast-dropout state. We tested also $33 \mathrm{~ns}$ and $37 \mathrm{~ns}$ and found that our results do not hinge upon the specific value of the threshold.

From the labeled ISI sequence one can determine the mean number, mean length and variance of ISIs within a fast- (slow-) dropout epoch, yielding values for $k_{1 / 2}, \lambda_{1 / 2}$ and $C_{\mathrm{V}, 1 / 2}$, respectively.

In fig. 3(b) we show $\rho_{n}$ for a constant pump level, within the range in which the correlation $\rho_{1}$ is maximal (fig. 2(a)). The solid lines represent the theoretical expression given in eq. (10), using the values determined from the analysis of the dropout series described above. Although the assumptions of our two-level theory are not fully obeyed, we observe a reasonable agreement, indicating that the main source for the correlations of the total sequence is an intrinsic switching between two states with different dropout statistics.

In order to test our interim conclusions that it is the switching between two states with different excitation rate that generates ISI correlations in a semiconductor laser with feedback, we now apply an external dichotomous modulation to the system, which we can control experimentally at will. Dichotomous noise has previously been found to lead to non-trivial correlations of residence times in bistable systems $[18,19]$. Here we consider that the input signal of our excitable laser switches between two values $\left(V_{1}\right.$ and $\left.V_{2}\right)$ with (symmetric) rate $\lambda$, and has an exponential correlation function $\left\langle\eta(t) \eta\left(t^{\prime}\right)\right\rangle=\sigma^{2} \exp \left[-2 \lambda\left|t-t^{\prime}\right|\right]$, corresponding to a correlation time $\frac{1}{2 \lambda}$. The length of the time series used to calculate the autocorrelation was $1 \mathrm{~ms}$. It is well known that the pumping of a semiconductor laser with feedback decreases the mean time between consecutive power dropouts, i.e. it increases the mean dropout rate of the excitable system. Due to the pump dependence of the dropout rate, the dichotomous driving leads to switching between two dropout rates, as shown in fig. 4(a). The bottom trace in the figure depicts the response of the laser to the dichotomous signal represented in the top trace, for a particular switching rate $\lambda$ and specific pump levels. Figure 4(b) compares the serial correlation coefficient at a pump of $32 \mathrm{~mA}$ for different lags with the theory described above. The correlation exhibits an exponential decay with a good agreement with the theoretical predictions given by eq. (10).

The controllability provided by the external dichotomous pump modulation allows us to vary the ratio of the dropout rates, $k_{2} / k_{1}$, over several orders of magnitude; (a)

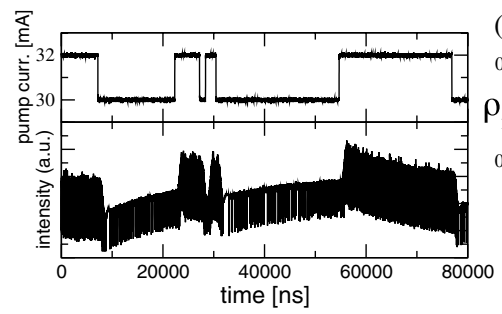

(b)

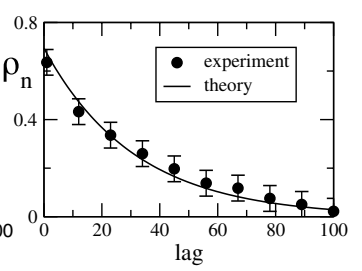

Fig. 4: (a) Symmetric dichotomous noise on the pumping current (top, $\lambda_{1,2}=\lambda=100 \mathrm{kHz}$ ) leads to a modulation of the dropout rates (bottom). (b) Correlation coefficient $\rho_{n}$ measured from the extracted interval sequence (symbols) and two-state theory (solid line), for which the parameters were determined from the unperturbed system.

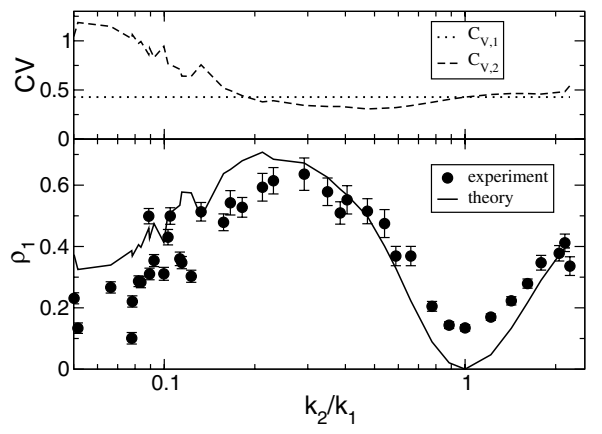

Fig. 5: CVs estimated from sets of DC experiments (upper panel) and correlation at lag 1 as a function of the firing-rate ratio $\gamma=k_{2} / k_{1}$ (circles). $k_{1}$ was held constant corresponding to a pump current of $32 \mathrm{~mA}$ (hence, $C_{V, 1}$ is constant), whereas $k_{2}$ was varied by varying the pump level of state 2 . The twostate theory (based on the measured rates and coefficients of variation of the respective unperturbed processes) is depicted by the solid line. Here $\hat{\lambda}_{1}=\hat{\lambda}_{2}=0.007$.

such a variation also causes a change in the CVs, see below. Figure 5 shows the first-order correlation coefficient $\rho_{1} v s$. that ratio (bottom panel) and the coefficients of variation of the two states, when the pump is applied continuously (top panel). Note that the CVs in the two cases are clearly non-zero, which highlights the noise-driven nature of the two states. The dropout rates are estimated as the inverse of the average ISI from time traces of the laser's output intensity when subject to constant pump levels corresponding to each of the two levels of the dichotomous modulation. In this experiment, we have kept one of the pumping levels of the dichotomous modulation constant (i.e. $k_{1}$ and $C_{V, 1}$ remain constant), and vary continuously the other one (i.e. changing $k_{2}$ and $C_{V, 2}$ ). The correlation shows a minimum (different from zero) when the dropout rates are equal (i.e. in the constant pump case); here our theory (which takes now into account only the externally imposed states) predicts a renewal point process for which $\rho_{1}=0$. As was shown above, the laser already generates a non-renewal dropout sequence on its own and hence in the experiment $\rho_{1} \neq 0$ for $k_{1}=k_{2}$. More importantly, the experimentally measured $\rho_{1}$ increases when $k_{2}$ departs 
from $k_{1}$ (as predicted by theory, see below). Interestingly, for $k_{2}<k_{1}$ the first-order correlation plotted $v s$. the firingrate ratio shows a non-monotonic behavior for decreasing pumping level $V_{2} \cdot \rho_{1}$ first grows as $k_{2}$ decreases away from $k_{1}$ (and $C_{V, 2} \approx C_{V, 1}$ ), reaches a maximum for an intermediate value of $k_{2}$, and decays again for very small $k_{2}$ (where $C_{V, 2}$ attains high values).

The non-monotonic behavior is qualitatively reproduced by the theoretical expression given in eq. (10), shown by the solid line in fig. 5. The theory has no free parameter and in this case has been applied to the external switching, instead of the internal one considered at the beginning of the paper. Note, that we use for the theoretical line the coefficient of variation $C_{V, 1}$ and $C_{V, 2}$ as measured in experiments with constant pump currents (that is why the theoretical curve for $\rho_{1}$ shows experimental jitter). In particular, in eq. (10) the parameter $C_{V, 2}$ can be regarded as a function of $k_{2}$. How much does the dependence of $C_{V, 2}$ on $k_{2}$ contribute to the shaping of the maximum in fig. 5 ? Our theory taken at constant values of the CVs would also predict a maximum, although a more shallow one and located at slightly smaller ratio ${ }^{1} k_{2} / k_{1}$, demonstrating that the decrease of $\rho_{1}$ at small $k_{2}$ in the bottom panel of fig. 5 is largely due to the increase of the $C_{V, 2}$ seen in fig. 5 .

In summary, we have shown experimentally that the power dropouts exhibited by a semiconductor laser with optical feedback display, for intermediate pump levels, correlations between interspike intervals, and have proposed a discrete kinetic model that interprets the mechanism of appearance of those correlations as resulting from the intrinsic switching of the system between two states with different dropout rates. In order to test this conclusion we have further applied an external random dichotomous modulation to the laser's pump level, and have observed that this external modulation also generates correlations in the time intervals between successive dropouts. The correlations under the dichotomous modulation exhibit a characteristic pattern as a function of the ratio between dropout rates of the two modulation levels, which agrees with analytical results obtained in the discrete kinetic model. These results shed light on a potential intrinsic cause of ISI correlations, which should be relevant to various spiking systems where ISI correlations are important, such as neuronal systems.

This research was supported in part by the Spanish Ministerio de Ciencia e Innovación through project
FIS2009-13360-C03-02, and by the Agència de Gestió d'Ajuts Universitaris i de Recerca (AGAUR), Generalitat de Catalunya, through project 2009 SGR 1168. JGO also acknowledges the ICREA foundation for financial support. TS and BL acknowledge support by the BMBF (FKZ: 01GQ1001A).

\section{REFERENCES}

[1] Livina V. N., Havlin S. and Bunde A., Phys. Rev. Lett., 95 (2005) 208501.

[2] Tiana-Alsina J., Torrent M. C., Rosso O. A., Masoller C. and Garcia-Ojalvo J., Phys. Rev. A, 82 (2010) 013819.

[3] Rubido N., Tiana-Alsina J., Torrent M. C., Garcia-Ojalvo J. and Masoller C., Phys. Rev. E, 84 (2011) 026202.

[4] Farkhooi F., Strube-Bloss M. F. and Nawrot M. P., Phys. Rev. E, 79 (2009) 021905.

[5] Avila-Akerberg O. and Chacron M. J., Exp. Brain Res., 210 (2011) 353.

[6] Chacron M. J., Longtin A. and Maler L., J. Neurosci., 21 (2001) 5328.

[7] Chacron M. J., Lindner B. and Longtin A., Phys. Rev. Lett., 92 (2004) 080601.

[8] Schwalger T., Fisch K., Benda J. and Lindner B., PLoS Comput. Biol., 6 (2010) e1001026.

[9] Sano T., Phys. Rev. A, 50 (1994) 2719.

[10] Fischer I., van TartwiJk G. H. M., Levine A. M., Els̈̈sser W., GöBel E. and Lenstra D., Phys. Rev. Lett., 76 (1996) 220.

[11] Giudici M., Green C., Giacomelli G., Nespolo U. and Tredicce J. R., Phys. Rev. E, 55 (1997) 6414.

[12] Mulet J. and Mirasso C., Phys. Rev. E, 59 (1999) 5400.

[13] Yacomotti A. M., Eguia M. C., Aliaga J., Martinez O. E. and Mindlin G. B., Phys. Rev. Lett., 83 (1999) 292.

[14] Cox D. and Lewis P., The Statistical Analysis of Series of Events (Chapman and Hall) 1966.

[15] Engel T. A., Schimansky-Geier L., Herz A., Schreiber S. and Erchova I., J. Neurophysiol., 100 (2008) 1576.

[16] Schwalger T. and Lindner B., Eur. Phys. J. ST, 187 (2010) 211.

[17] Gardiner C. W., Stochastic Methods: A Handbook for the Natural and Social Sciences (Springer) 2009.

[18] Lindner B. and Schwalger T., Phys. Rev. Lett., 98 (2007) 210603.

[19] Schwalger T. and Lindner B., Phys. Rev. E, 78 (2008) 021121.

\footnotetext{
${ }^{1}$ To obtain an estimate of the maximizing ratio, we set for simplicity the switching rates of the two states equal to $\hat{\lambda}$, and assume equal coefficients of variations $C_{V}=C_{V, 1}=C_{V, 2}$, that do not depend on $\gamma=k_{2} / k_{1}$. For $\hat{\lambda} \ll 1$, we find that $\gamma_{\max } \approx \sqrt{\hat{\lambda}\left(1+1 / C_{V}^{2}\right)}$. For the data in fig. 5 with $\hat{\lambda} \approx 0.007$ and an average $C_{V} \approx 0.3$, we find from our formula $k_{2, \text { max }} \approx 0.15 k_{1}$, which is a reasonable estimate of $0.2 k_{1}$ (full theory) and $0.3 k_{1}$ (experiment) in fig. 5 .
} 021121 